\title{
Extramandibular Presentation in the Course of the Inferior Alveolar Nerve: A Case Report and Review of the Literature
}

\author{
Ilson Sepulveda ${ }^{\mathrm{a}, \mathrm{b}, \mathrm{c}, \mathrm{d}, \mathrm{h}, \mathrm{J} \text {. Patricio Ulloa }}{ }^{\mathrm{d}, \mathrm{e}}$, Francisco Rivas-Rodriguez ${ }^{\mathrm{f}}$, \\ Rodrigo Barros ${ }^{\mathrm{g}}$
}

\begin{abstract}
The inferior alveolar nerve (IAN) is a branch of the mandibular nerve, the third division of the trigeminal nerve ( $\mathrm{V}$ cranial nerve). Its location and course into the mandible is very important because of its susceptibility to the injury during any surgical or prosthetic approaches. Cone beam computed tomography (CBCT) is a three-dimensional imaging modality, with less exposure than multi slice computed tomography (MSCT), being considered an ideal imaging tool for maxillofacial diagnosis. We present an unusual extramandibular submucosal course of IAN in relationship with a mandibular exostosis, and with no association with the sensory disorder.
\end{abstract}

Keywords: Atrophic; Mandible; Exostosis; Incidental finding; Cone beam computed tomography

\section{Introduction}

The inferior alveolar nerve (IAN) enters to the mandible by the mandibular foramen, located on medial aspect of the ramus, and runs in anterior and lateral direction inside the mandibular body, until exits by the mental foramen at the premolar region. In this course, it gives sensory nerves from incisive (through incisive nerve) to molar region, including to the mentolabial area. The knowledge of its considerable variation

Manuscript submitted November 13, 2018, accepted November 21, 2018

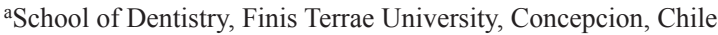

${ }^{b}$ Radiology Department, ENT-Head and Neck Surgery, Concepcion, Chile

cDental-Maxillofacial and Nuclear Medicine Services, General Hospital of Concepcion, Concepcion, Chile

${ }^{\mathrm{d}}$ ENT-Head and Neck Surgery Service, General Hospital of Concepcion, Concepcion, Chile

eUniversity of Concepcion School of Medicine, Concepcion, Chile

fDivision of Neuroradiology, Radiology Department, University of Michigan Health System, Ann Arbor, MI 48109, USA

'Emergency and Internal Medicine Services, General Hospital of Concepcion, Concepcion, Chile

${ }^{\text {h} C o r r e s p o n d i n g ~ A u t h o r: ~ I l s o n ~ S e p u l v e d a, ~ E N T-H e a d ~ a n d ~ N e c k ~ S u r g e r y ~ S e r-~}$ vice, General Hospital of Concepcion, San Martin Av. 1436, Concepcion, Chile. Email: isepulvedaa@uft.edu

doi: https://doi.org/10.14740/jmc3215 in the course, shape, curve, and directions is essential at moment to any surgical procedure plan in this area. Cone beam computed tomography $(\mathrm{CBCT})$ provides an accurate location, and to determine any anatomical variation in the course of the mandibular canal and its relation with neighbor anatomical structures $[1,2]$.

\section{Case Report}

A 75-years-old female without a significant medical history, presented for imaging study of swelling on the medial aspect of the right mandibular body. The clinical exam revealed total edentulous jaw and hard swelling in the area mentioned above, without ulceration, pain or paresthesia. No other injuries or cervical lymphadenopathy were identified. Also, the patient presented no other symptoms.

CBCT was performed showing a hyperdense exostosis of the cortical bone without compromise of the bone marrow. As incidental finding demonstrated the entry and exit of the IAN canal in relationship with the exostosis (Fig. 1a, b), the volume rendering with virtual representation of the IAN confirmed this finding (Fig. 1c, d).

\section{Discussion}

IAN located outside of the mandible is a rare condition; it's the most common presentation which is associated to severe atrophy in edentulous mandibles or hypoplastic condition in dentulous mandible [1]. Various imaging modalities including the conventional and computerized tomography have been used to evaluate mandibular morphology before a surgical procedure. In CBCT the appearance of the mandibular canal usually is a well-defined hypodense zone, lined by hyperdense borders [2].

From its origin, IAN runs obliquely down and forward, enters the medial mandibular ramus through the mandibular foramen; runs more or less horizontal, and ends between the apices of the first and second premolar, exiting the mental foramen giving terminal mental and incisive nerves [3].

Worthington described that there are three different configurations of the inferior alveolar canal (IAC) as follows: 1) A progressive curve rising from anterior to posterior; 2) A steep ascent from anterior to posterior; and 3) A catenary-like canal [4].

Sanam M. et al evaluated the IAN course in three age 


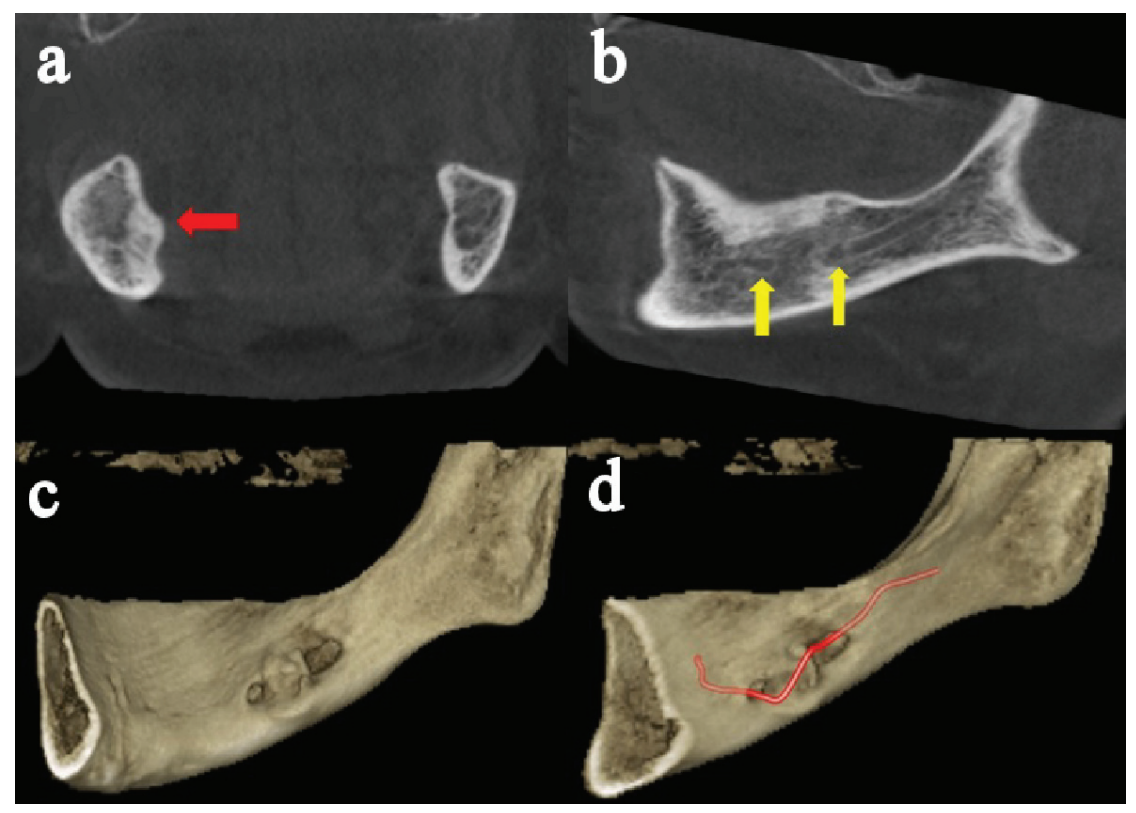

Figure 1. СBCT of the mandible shows a cortical bone exostosis in medial aspect of the right mandibular body (red arrow), exit and entry holes of the mandibular canal (yellow arrows). As shown the three- dimensional IAN course representation (red). (a) Coronal. (b) Oblique sagittal. (c, d) Osseous volume rendering.

groups and demonstrated that straight and progressive descending pattern were the most prevalent pattern in the 20 - 29 years age group. In the $30-44$ and 45 - 59 years age group the most prevalent category was recorded as straight and catenary, respectively, without any statistically significant difference between the three age groups [5].

Therefore, information on the buccolingual, superior and inferior position of the canal could assist surgeons in avoiding injury to the IAN [6]. Atieh Safaee et al established that the greatest distance, from IAC to the buccal cortex existed at the second molar region, where the lingual distances in some cases reached zero [7]. In the vertical dimension, A. Ozturk et al demonstrated that on average, the IAC rans almost $1 \mathrm{~cm}$ (range $6.10-15.30 \mathrm{~mm}$ ) above the inferior border of the body of the mandible in the molar region [8].

Extracanalicular variations of the IAN are exceedingly rare and unreported. R. Manikandhan et al reported absent mandibular canal and mental foramen associated to unilateral hemifacial microsomia, including hypoplasia of the facial musculature with a mild degree of microtia [9]. C. Herlin et al reported a very short course in the left IAC, opening onto the lateral surface of the mandibular angle. There was no other foramen on the lateral wall of the left mandibular body. No morphological abnormality of the face was noted on clinical examination [10].

\section{Conclusions}

Better understanding of the anatomic variation of the nerve may alter the planning of surgical procedures. Based on anatomical features and imaging findings, potential damage of the IAN during oral and dental operative treatment procedures in this region can be alleviated.

\section{Financial Support}

None.

\section{References}

1. Rusu MC, Sandulescu M, Ciuluvica RC, Sendroiu VM, Didilescu AC. The extramandibular inferior alveolar nerve in cases with severely atrophic lower jaws. Surg Radiol Anat. 2012;34(3):277-279.

2. Balaji SM, Krishnaswamy NR, Kumar SM, Rooban T. Inferior alveolar nerve canal position among South Indians: A cone beam computed tomographic pilot study. Ann Maxillofac Surg. 2012;2(1):51-55.

3. Yeh AYE, Finn BP, Jones RHB, Goss AN. The variable position of the inferior alveolar nerve (IAN) in the mandibular ramus: a computed tomography (CT) study. Surg Radiol Anat. 2018;40(6):653-665.

4. Worthington P. Injury to the inferior alveolar nerve during implant placement: a formula for protection of the patient and clinician. Int J Oral Maxillofac Implants. 2004;19(5):731-734.

5. Mirbeigi S, Kazemipoor M, Khojastepour L. Evaluation of the course of the inferior alveolar canal: the first CBCT study in an Iranian population. Pol J Radiol. 2016;81:338341 .

6. Khorshidi H, Raoofi S, Ghapanchi J, Shahidi S, Paknahad M. Cone beam computed tomographic analysis of the course and position of mandibular canal. J Maxillofac 
Oral Surg. 2017;16(3):306-311.

7. Safaee A, Mirbeigi S, Ezoddini F, Khojastepour L, Navab-Azam A. Buccolingual course of the inferior alveolar canal in different mental foramen locations: A cone beam computed tomography study of an Iranian population. Int J Appl Basic Med Res. 2016;6(4):262266.

8. Ozturk A, Potluri A, Vieira AR. Position and course of the mandibular canal in skulls. Oral Surg Oral Med Oral
Pathol Oral Radiol. 2012;113(4):453-458.

9. Manikandhan R, Mathew PC, Naveenkumar J, Anantanarayanan P. A rare variation in the course of the inferior alveolar nerve. Int J Oral Maxillofac Surg. 2010;39(2):185187.

10. Herlin C, Yachouh J, Journet JL, Goudot P. Extramandibular position of the inferior alveolar nerve. Analysis and review of the literature. Surg Radiol Anat. 2013;35(9):811815. 